\title{
Radioterapia extereotáxica extracraneal
}

P. Muto

\section{Introduction}

Small-volume radiotherapy, which uses a small planning target volume (PTV) margin either with a single dose or hypo fractionation, is widely used for metastatic brain tumours. Stereotactic radiosurgery or hypofractionated radiotherapy shows an $80-90 \%$ local control rate, even for so-called radio resistant tumours such as renal cell carcinoma and melano$\mathrm{ma}^{1,2}$. Because of its success, small-volume radiotherapy has been applied to extracranial lesions, such as lung and liver tumours, using various techniques ${ }^{3-5}$. Although for patients with Stage I non-small-cell lung cancer (NSCLC), surgery is the primary treatment of choice, radiotherapy is often indicated if the patients have a medically inoperable condition such as poor pulmonary or cardiac function. The results of conventional radiotherapy for Stage I lung cancer, however, are not satisfactory. Five-year actuarial survival of convention radiotherapy ranged from $6 \%$ to $27 \% 6-9$, which was unsatisfactory compared with surgery, which has a 5 -year survival rate of $60 \%$ to $80 \%$, even considering that poor-risk patients receive the radiotherapy series ${ }^{10}$. Because some studies reported that patients with lung cancers who received high-dose radiation $^{10-12}$ and those who received a high dose in a short period $^{10}$ survived for a longer period, dose escalation in a short treatment time may improve the survival rate for NSCLC patients $^{13-15}$

Metastatic lung cancer is common in cancer patients, and its prognosis is usually poor. But some patients with slow-growing tumours survive for longer periods ${ }^{16}$. These patients are sometimes managed with surgical resection of the metastatic lesions to reduce the possibility of symptomatic complications and to prolong their symptom-free status.

In 1995, Blomgren et al. introduced a new stereotactic treatment technique for extracranial radiotherapy that was analogous to radiosurgery in the brain ${ }^{38}$. The treatment of liver and lung tumours seemed very promising, leading to superior local control, with only 1 recurrence in 40 treated tu-

Radioterapia Villa del Sole Napoli (Italia) mours. Based on these data, in 1997 we started a Phase I/II study for focused stereotactic radiosurgery of localized liver and lung tumors, though, in contrast to Blomgren et al. ${ }^{38}$, strictly pursuing a single-dose concept ${ }^{39}$. It was shown that precise patient positioning and tumour stabilization could be achieved, allowing for high single-dose treatment.

\section{Materials and methods}

Between November 2001 and December 2003, 60 patients affected by a primitive or recurrent lung cancer or by lung metastases were treated with hypofractionated stereotactic arc radiotherapy. Radiotherapy was delivered with a 6-15 Mv linear Accelerator using an arc technique with a micro-multi-leaf device. Totally we treated 71 lung tumours, with a total number of 278 fractions of stereotactic body radiosurgery. Of these patients 15/60 had a lung metastasis by a distal cancer (5 from breast, 5 rectum, 3 larynx, 1 kidney, 1 melanoma), 17/60 were lung metastases from a NSCLC, 18 were primitive lung cancers (NSCLC) al 10 were local recurrences of NSCLC.

Of these patients $17 / 60$ received also a treatment with external beam radiotherapy (EBRT) previously, and 32/60 were also treated with chemotherapy.

Some of the patients had multiple lesions, but the majority $(51 / 60)$ had a single lesion. There were 4 patients with 3 lung metastases each and 5 patients with 2 . Some (17/60) had also non pulmonary metastasis ( 3 brain, 5 liver, 3 surrenal gland, 10 bone). Four patients had multiple lesions.

The median diameter of the treated lesions was of $2,5 \mathrm{~cm}$ (range $0,7-5 \mathrm{~cm}$ ).

The prescribed dose per fraction ranged between 4 Gy and 10 Gy (10Gy in 10 treatments, 9 Gy in 52 treatments, and a dose lower than $9 \mathrm{~Gy}$ in 9 treatments).

The prescribed total dose ranged between 30Gy and $40 \mathrm{~Gy}$ ( $30 \mathrm{~Gy}$ in 2 treatments, 32Gy in 3, 36Gy in 56, 40gy in 10).

The treatments lasted between 11 and 26 days each.

The number of fractions ranged between 4 and 8 for each treatment (4 fractions in 68, 6 fractions in 2, 8 fractions in 1). The number of arcs for each therapy ranged between 3 and 7 . In the majority it was delivered with 4 or 5 arcs. More than $90 \%$ of the PTV was delivered the prescribed dose in the majority of cases (average; $96 \%$, range; $74-100 \%$ ). Two pre- 
requisites for stereotactic high-dose therapy are accurate target localization and high levels of geometrical accuracy in dose delivery. The most outstanding problem in stereotactic radiosurgery for thoracic tumors is respiratory motion. Several studies investigating the amplitude of respiration-related organ motion yielded a maximum value of approximately 2 $\mathrm{cm}$ in the cranio-caudal direction with normal breathing. There are two major methods to reduce the uncertainty caused by respiratory motion. One is reduction of the breathing motion with pressure that is represented by the stereotactic body frame. The other is the respiration-gated intermittent irradiation system. We had very satisfactory results using a stereotactic body frame plus a vack-lock device. Set-up error was within $5 \mathrm{~mm}$ in all directions (X, Y, Z axis).

\section{Results}

The median follow up is of 9 months (range 1-24 months).

Patients were seen and examined by one or two of the investigating physicians a minimum of once per month in the first 6 months of the follow-up period and every 3 months thereafter. This evaluation included a physical examination, laboratory evaluation, and chest CT scan. A respiratory function test was obtained in patients who agreed to do it regularly during the follow-up.

We obtained an improvement of symptoms with a regression of the respiratory distress in 16 patients $(25 \%)$ and a reduction of the volume valuable at the CT scan in 32 patients (50\%).

In 20 patients (33\%) we obtained a RP, in 25 (41\%) a stable disease (SD), in $15(25 \%)$ it was diagnosed a local progression of the tumor.

Totally 15 patients died for the consequences of the extrapulmonary disease (7 of these had also a local progression).

In 27/60 patients it was described an asymptomatic lung fibrosis medially 4 months after treatment. Only 5/60 patients had an increased dyspnoea.

\section{Discussion}

In some experiences with hypofractionated stereotactic radiosurgery 40 the 3 -year local control rate was $80.4 \% \pm 7.1 \%$ (a standard error) with a median follow-up period of 17 months for survivors. The 3-year local control rate was 69.6 $\pm 10.6 \%$ for patients who received 48 Gy and $100 \%$ for patients who received $60 \mathrm{~Gy}$.

But in this series because of the Grade 5 toxicity, they halted this Phase I/II study and planned to rearrange the protocol setting. We didn't recorded such side effects

Organ movement is an important issue as a source of uncertainty in our study. Systematic or preparatory error resulting from organ motion can significantly increase or decrease the dose distribution for the critical organs in general ${ }^{32}$. Intrafractional organ motion can affect dose distribution considerably for lung tissues. Breath-holding, gating, and respiratory synchronization have been used to reduce the uncertainty resulting from respiratory movement ${ }^{33-37}$. Technical developments may effectively reduce the safety margin for internal motion, but their clinical importance should be compared carefully with the technique introduced in the current study because our method does not require investment in new equipment.

Stereotactic treatment of lung tumours is described by some authors. Nakagawa et al. ${ }^{44}$ treated 22 thoracic tumors with single doses between 15 and 24 Gy. During a median follow-up of 8 months, only 1 local recurrence was seen. A concept of hypofractionated treatment in up to 3 treatment sessions was pursued by Blomgren et al. ${ }^{45}$ in most of the 17 irradiated lung tumors. Sixteen of these presented at least stable disease in the mean follow-up period of 8.2 months. A similar approach was taken by Wulf et al. ${ }^{46}$, achieving $76 \%$ actuarial local control after 2 years in 27 treated lung tumours. Even smaller fraction-doses (total dose: 50-60 Gy in 5-10 fractions) were used by Uematsu et al. ${ }^{47}$ for the treatment of Stage I NSCLC. In a relatively large group of 50 patients with a long median follow-up of 36 months, local tumour control was achieved in $94 \%$ of the patients. Yet, it has to be mentioned that 18 patients had received conventional radiotherapy earlier.

According to these encouraging results, our own data seem to be comparable given the small patient numbers in most of the studies presented, while offering the advantage of reasonable time-effort with the single-fraction treatment.

Optimal indication of this technique is controversial. Although its local control rate for metastatic thoracic tumors is favorable, most of them, small lung metastases in particular, are symptom free. This point is contrary to the symptomatic brain metastasis and may limit clinical value of the treatment. However, solitary metastases that appear during long followup may be suitable for it. Furthermore, several previous papers reported encouraging results with limited field radiation therapy for early-stage (T1-2N0) non-small-cell peripheral lung cancer.

\section{References}

1. Alexander 3rd E, Moriarty TM, Davis RB, et al. stereotactic radiosurgery for the definitive, non-invasive treatment of brain metastases. J Natl Cancer Inst 1995; 87:34-40.

2. Shirato $H$, Takamura A, Tomita $M$, et al. stereotactic irradiation without whole-brain irradiation for single brain metastasis. Int J Radiat Oncol Biol Phys 1997; 37:385-91.

3. Blomgrem H, Lax I, Naslund I, et al. stereotactic high dose fraction radiation therapy of extracranial tumors using an accelerator. Clinical experience of the first thirty-one patients. Acta Oncol 1995; 34:861-70.

4. Herfarth KK, Debus J, Lohr F. stereotactic single-dose radiation therapy of liver tumors: Results of a phase I/II trial. J Clin Oncol 2001; 19:164-70.

5. Uematsu M, Shioda A, Suda A, et al. Computed tomographyguided frameless stereotactic radiotherapy for stage I non-small cell lung cancer: A 5-year experience. Int J Radiat Oncol Biol Phys 2001; 51:666-70.

6. Mori Y, Kondziolka D, Flickinger JC, et al stereotactic radiosurgery for cerebral metastatic melanoma: factors affecting local disease control and survival. Int J Radiat Oncol Biol Phys 1998; 42:581-9

7. Morita K, Fuwa N, Suzuki Y, et al. Radical radiotherapy for medically inoperable non-small cell lung cancer in clinical stage I: A retrospective analysis of 149 patients. Radiother Oncol 1997; 42:31-6. 


\section{P. Muto}

8. Cheung PC, Mackillop WJ, Dixon P, et al. Involved-field radiotherapy alone for early-stage non-small-cell lung cancer. Int J Radiat Oncol Biol Phys 2000; 48:703-10.

9. Gauden S, Ramsay J, Tripcony L. The curative treatment by radiotherapy alone of stage I non-small cell carcinoma of the lung. Chest 1995; 108:1278-82.

10. Slotman BJ, Nio KH, Karim AB. Curative radiotherapy for technically operable stage I nonsmall cell lung cancer. Int J Radiat Oncol Biol Phys 1994; 29:33-7.

11. Kaskowitz L, Graham MV, Emami B, et al. Radiation therapy alone for stage I non-small cell lung cancer. Int J Radiat Oncol Biol Phys 1993; 27:517-23.

12. Sherman DM, Weichselbaum R, Hellman $S$. The characteristics of long-term survivors of lung cancer treated with radiation. Cancer 1981; 47:2575-80.

13. Maguire PD, Marks LB, Sibley GS, et al. 73.6: Gy beyond: Hyperfractionated, accelerated radiotherapy for non-small-cell lung cancer. J Clin Oncol 2001; 19:705-11.

14. Dominioni L, Imperatori A, Rovera F, et al. Stage I nonsmall cell lung carcinoma. Analysis of survival and implications for screening. Cancer 2000; 89(Suppl. 11):2334-44.

15. Hayman JA, Martel MK, Haken RKT, et al. Dose escalation in non-small cell lung cancer using three-dimensional conformal radiation therapy: Update of a phase I trial. J Clin Oncol 2001; 19:127-36.

32. van Herk $M$, Remeijer $P$, Lebesque JV. Inclusion of geometric uncertainties in treatment plan evaluation. Int J Radiat Oncol Biol Phys 2002; 52:1407-22.

33. Hanley J, Debois MM, Mah D, et al. Deep inspiration breathhold technique for lung tumors: The potential value of target immobilization and reduced lung density in dose escalation. Int J Radiat Oncol Biol Phys 1999; 45:603-11.

34. Halperin $R$, Roa $W$, Field $M$, et al. Setup reproducibility in radiation therapy for lung cancer: A comparison between T-bar and expanded foam immobilization divides. Int J Radiat Oncol Biol Phys 1999; 43:211-6.

35. Lohr F, Debus J, Frank C, et al. Noninvasive patient fixation for extracranial stereotactic radiotherapy. Int J Radiat Oncol Biol Phys 1999; 45:521-7.

36. Tada $T$, Minakuchi $K$, Fujioka $T$, et al. lung cancer: Intermittent irradiation synchronized with respiratory motion: Results of a pilot study. Radiology 1998; 207:779-83.
37. Shirato H, Shimizu S, Kitamura K, et al. Four-dimensional treatment planning and fluoroscopic real-time tracking radiotherapy for moving tumor. Int J Radiat Oncol Biol Phys 2000; 48:43542.

38. Calvo FA, Samblas J, Santos M, Delgado JM. Stereotactic radiosurgery with linear accelerator. Rays 1998 Jul-Sep; 23(3):462-85.

39. Herfarth KK, Lohr F, Bahner ML, et al. stereotactic single dose radiation therapy of tumors in the lung. Radiology 2000; 217(Suppl.): 148.

40. Lee SW, Choi EK, Park HJ, Ahn SD, Kim JH, Kim KJ, Yoon SM, Kim YS, Yi BY. Stereotactic body frame based fractionated radiosurgery on consecutive days for primary or metastatic tumors in the lung. Lung Cancer 2003 Jun; 40(3):309-15.

41. Hadinger U, Thiele W, Wulf J. Extracranial stereotactic radiotherapy: evaluation of PTV coverage and dose conformity. Z Med Phys 2002; 12(4):221-9.

42. Cardinale RM, Wu Q, Benedict SH, Kavanagh BD, Bump E, Mohan R. Determining the optimal block margin on the planning target volume for extracranial stereotactic radiotherapy. Int J Radiat Oncol Biol Phys 1999 Sep 1; 45(2):515-20.

43. Rikiya Onimaru, Hiroki Shirato, Shinichi Shimizu, Kei Kitamura, B.o Xu, Shin-ichi Fukumoto, T.a-Chen Chang, Katsuhisa Fujita, Masataka Oita, Kazuo Miyasaka, Masaharu Nishimura and Hirotoshi Dosaka-Akita. Tolerance of organs at risk in small-volume, hypofractionated, image-guided radiotherapy for primary and metastatic lung cancers. Int J Radiat Oncol Biol Phys 2003 Volume 56, Issue 1, Pages 126-35.

44. Nakagawa K, Aoki Y, Tago M, et al. Megavoltage CT-assisted stereotactic radiosurgery for thoracic tumors: Original research in the treatment of thoracic neoplasms. Int J Radiat Oncol Biol Phys 2000; 48:449-57.

45. Blomgren $\mathrm{H}$, Lax I, Göranson $\mathrm{H}$, et al. Radiosurgery for tumors in the body: Clinical experience using a new method. J Radiosurg 1998; 1:63-74.

46. Wulf J, Hädinger $U$, Oppitz $U$, et al. stereotactic radiotherapy of targets in the lung and liver. Strahlenther Onkol 2001; 177:645-55

47. Uematsu $M$, Shioda A, Suda A, et al. Intrafractional tumor position stability during computed tomography (CT)-guided frameless stereotactic radiation therapy for lung or liver cancers with a fusion of CT and linear accelerator (FOCAL) unit. Int J Radiat Oncol Biol Phys 2000; 48:443-8. 